Revista Facultad 2019

\title{
¿Qué influye en la confianza en las instituciones? Evidencia empírica para Chile*
}

\author{
Fabián Riffo ${ }^{\mathbf{a}}$ - Daniela Pérez ${ }^{\mathbf{b}}$ - César Salazarc - Andrés Acuñad
}

Resumen: Esta investigación estima un modelo probit ordenado con datos de la encuesta LAPOP de 2014, para explorar los factores que explican la confianza institucional en Chile. Los resultados indican mayor desconfianza desde los pueblos originarios hacia instituciones de seguridad, probablemente por el conflicto mapuche. Asimismo, se encuentra un efecto positivo de variables de democracia y desempeño, lo que revela diferencias en responsabilidades entre el ejecutivo y las municipalidades. Los resultados también dan soporte a la importancia de la participación ciudadana en los niveles de confianza y una evaluación positiva de las fuerzas armadas ante escenarios de corrupción. Finalmente, se evidencia que las personas a favor del aborto legal poseen niveles de confianza menores en la iglesia evangélica, lo cual puede ser atribuido a una posición más conservadora de esta religión.

Palabras clave: Capital social; confianza; instituciones; probit ordenado.

Fecha de recibido: 10 de junio de 2018 Fecha de aprobado: 21 de mayo de 2019

Cómo citar: Riffo, F., Pérez, D., Salazar, C. y Acuña, A. (2019). ¿Qué influye en la confianza en las instituciones? Evidencia empírica para Chile. Revista Facultad de Ciencias Económicas, 27(2), 83-104. rev. fac.cienc.econ. doi: https://doi.org/10.18359/rfce.3517

JEL: C35, P48.

* Artículo de investigación.

a Investigador del Departamento de Economía y Finanzas, Facultad de Ciencias Empresariales, Universidad del Bío-Bío, Concepción, Chile. E-mail: fariffo@alumnos.ubiobio.cl

b Investigador/a del Departamento de Economía y Finanzas, Facultad de Ciencias Empresariales, Universidad del Bío-Bío, Concepción, Chile. E-mail: dpereza@alumnos.ubiobio.cl

c PhD in Economics y académico del Departamento de Gestión Empresarial, Facultad de Ciencias Empresariales, Grupo de investigación Análisis Económico Sectorial Aplicado y Centro de Estudios de Ñuble, Universidad del Bío-Bío, Chillán, Chile. E-mail: csalazar@ubiobio.cl. Autor para correspondencia: Avenida Andrés Bello 720, Casilla 447, Código Postal 3800708, Chillán, Chile.

d Magíster en Economía y académico del Departamento de Economía y Finanzas, Facultad de Ciencias Empresariales y Grupo de investigación Análisis Económico Sectorial Aplicado, Universidad del Bío-Bío, Concepción, Chile. E-mail: aacunad@ubiobio.cl 


\title{
What has an Influence on Confidence in institutions? Empirical Evidence for Chile
}

\begin{abstract}
This research estimates an ordered probit model with data from the 2014 LAPOP survey to explore the factors that explain confidence in institutions in Chile. Results show an increased lack of confidence from the original peoples toward security institutions, probably due to the Mapuche Conflict. There is also a positive effect of democracy and performance variables that unveils differences in responsibilities between the executive power and the municipalities. Results also support the importance of citizen participation for levels of confidence and a positive assessment of the armed forces in corruption scenarios. Finally, it is evidenced that people in favor of legal abortion have lower levels of confidence in the evangelical church, which may be attributed to a more conservative stance in this religion.
\end{abstract}

Keywords: Social capital; confidence; institutions; ordered probit.

\section{O que influencia a confiança nas instituições? Evidência empírica para o Chile}

Resumo: Esta pesquisa estima um modelo de probit ordenado com dados da Pesquisa de Opinião Pública da América Latina (LAPOP, na sigla em inglês), de 2014, para explorar os fatores que explicam a confiança institucional no Chile. Os resultados indicam maior desconfiança dos povos originários em relação às instituições de segurança, provavelmente devido ao conflito mapuche. Também há um efeito positivo das variáveis de democracia e desempenho, que revela diferenças de responsabilidades entre o executivo e os municípios. Os resultados também apoiam a importância da participação dos cidadãos nos níveis de confiança e uma avaliação positiva das forças armadas diante dos cenários de corrupção. Finalmente, é evidente que as pessoas a favor do aborto legal têm níveis mais baixos de confiança na igreja evangélica, o que pode ser atribuído a uma posição mais conservadora dessa religião.

Palavras-chave: Capital social; confiança; instituições; probit ordenado. 


\section{Introducción}

La confianza institucional es un factor clave para la sociedad y unos de los requisitos fundamentales para una buena gobernanza en pos del desarrollo económico (Putnam, Leonardi y Nanetti, 1993). Como indicador de cohesión social, la confianza institucional es crucial en el diseño y la implementación de reformas económicas procrecimiento orientadas a incrementar la calidad de vida de los ciudadanos (Easterly, Ritzen y Woolcock, 2006). En efecto, altos niveles de confianza facilitan la provisión de bienes públicos y promueven la constitución de actores sociales y sociedades civiles saludables, lo que contribuye al desarrollo económico y social de un país (Aker, 2007; Knack y Keefer, 1997; Kliksberg, 1999; Horváth, 2013) y al bienestar subjetivo de sus habitantes (Fukuyama, 1995; Hudson, 2006; Hommerich, 2012). América Latina es la región del mundo donde existe mayor desconfianza institucional (Easterly et al., 2006; Proyecto de Opinión Publica en América Latina [LAPOP, por sus siglas en inglés], 2010), razón por la cual se convierte en una de las principales problemáticas de los Gobiernos. Los niveles de confianza institucional en Chile también presentan niveles bajos. De acuerdo con cifras entregadas por el Consejo de Transparencia (2017), se evidencia una baja confianza en las instituciones públicas, con valores que no superan el $30 \%$. Lo anterior revela una disconformidad generalizada con los organismos que representan a la población, lo que dificulta su capacidad para canalizar el pensar colectivo. Estas cifras se encuentran en línea con una marcada tendencia a la desafección política e insatisfacción con el funcionamiento de la democracia en Chile, evidenciada por una baja participación cívica y organizacional, y potenciada por los hechos de corrupción que se han revelado en los últimos años.

Esta investigación tiene como objetivo explorar los factores clave que influyen en los altos niveles de desconfianza institucional observados en Chile, con los datos proporcionados por la encuesta AmericasBarometer, aplicada en 2014 por el LAPOP, haciendo énfasis en factores socioeconómicos, de percepción sobre la democracia, de desempeño institucional, participación civil y corrupción.
Estudios previos han abordado esta problemática para América Latina y Chile; por ejemplo, empleando la encuesta LAPOP 2004, Morris y Klesner (2010) validaron la relación positiva entre la confianza interpersonal y la confianza institucional en México, controlando la endogeneidad provocada por la percepción y tolerancia a la corrupción en dicho país. Stoyan, Niedzwiecki, Morgan, Hartlyn y Espinal (2016) confirmaron el efecto positivo que el desempeño del Gobierno y el interés en la política generan sobre la confianza institucional en Haití y en República Dominicana, a pesar de las disimilitudes entre ambos países en términos de desarrollo. Beramendi, Delfino y Subierta (2016) evaluaron la relación entre la confianza institucional y la confianza social en el contexto argentino para una muestra de estudiantes, y encontraron así una reducción de los niveles de confianza en la policía y el Gobierno cuando los ciudadanos perciben un mal funcionamiento de las instituciones. En Chile, Morales (2008) evaluó de forma empírica la relación entre la democracia y la confianza institucional, utilizando los datos de la encuesta LAPOP; gracias a esto, encontró que la insatisfacción con la democracia reduce la confianza institucional principalmente en el Gobierno, en el Congreso y en los partidos políticos. De igual modo, Segovia, Haye, González, Manzi y Carvacho (2008), a partir de una encuesta aplicada en Santiago de Chile en 2005, evidenciaron el efecto positivo de la percepción ciudadana de la capacidad para ejercer funciones y la benevolencia sobre la confianza en organismos vinculados a los poderes del Estado y de orden y seguridad. Siguiendo estos trabajos, esta investigación estudia diferencias en la confianza institucional para un conjunto de instituciones enmarcadas en el plano judicial y de seguridad, político y legislativo, ejecutivo y Gobiernos locales e instituciones de carácter religioso. A diferencia de los estudios previos para América Latina, y considerando la naturaleza ordenada de la variable dependiente, este trabajo propone la estimación de un modelo probit ordenado para explicar los niveles de confianza institucional. El estudio se estructura de la siguiente manera: primero se presenta el marco conceptual del capital social, confianza institucional y sus factores clave; luego, la 
metodología y los datos utilizados en este estudio. Seguido de esto, se discuten los resultados y por último se presentan las conclusiones del estudio.

\section{Marco conceptual}

\section{Capital social y confianza institucional}

Coleman (1988) define el capital social como los "aspectos de las organizaciones sociales, tales como las redes, las normas y la confianza que permiten la acción y la cooperación para el beneficio mutuo" (p. 98). Por su parte, Putnam et al. (1993) agregan que el capital social puede "mejorar la eficiencia de la sociedad mediante la facilitación de las acciones coordinadas" (p. 167). Lo anterior sugiere que el capital social, al fortalecer las instituciones, puede tener una incidencia positiva en el crecimiento y desarrollo económico (Granovetter, 1985; Wilson, 1996; Kliksberg, 1999; Woolcock y Narayan, 2000; Ahlerup, Olsson, Yanagizawa, 2009; Westlund y Adam, 2010).

Uno de los elementos clave del capital social es la confianza; desde el punto de vista individual, esta última es "una hipótesis sobre la conducta futura de otras personas, en la medida en que mis resultados dependen de la acción de estos" (Cornú, 1999, p. 19). Por otra parte, Mayer, Davis y Schoorman (1995) sostienen que "la confianza puede ser vista como la buena voluntad de una persona de ser vulnerable a las acciones de otra" (p. 712). Altos niveles de confianza son la base para promover los principios de cooperación entre los agentes sociales, lo que posibilita formas eficientes de organizaciones económicas que reducen los costos de transacción y negociación, con impactos positivos en el desempeño de las instituciones, resolución de conflictos de bienes públicos y crecimiento económico (Coleman, 1988; Fukuyama, 1995; Gordon, 2006; Horváth, 2013).

Reconociendo el rol de la confianza individual en los demás, Knight (1998) releva además la importancia de la confianza permanente y generalizada en la sociedad como un todo. Lo anterior se relaciona con la confianza en el entorno institucional, el cual favorece el cumplimiento de las leyes y normas sociales. Las instituciones son el nexo entre la ciudadanía y las autoridades, y funcionan como un puente de interacción entre estos (Luhmann, 2005).

De acuerdo con Easton (1965), la confianza hacia las instituciones puede ser entendida como una forma de apoyo "difuso" al régimen político expresado en actitudes favorables a la democracia, o como un respaldo "específico" según el desempeño y los resultados alcanzados. Por otra parte, la literatura concibe la confianza institucional como una métrica de cohesión social. Para Easterly et al. (2006) el grado de cohesión social, determinada por la confianza hacia las instituciones, contribuye al fortalecimiento y a la calidad de estas.

\section{Factores clave de la confianza institucional}

Uno de los factores relevantes que condiciona la confianza en las instituciones se relaciona con la satisfacción y percepción de la democracia. Almond y Verba (2015) sostienen que la democratización inspira la creación de instituciones para el empoderamiento ciudadano, siendo este el mecanismo mediante el cual decantan todas las posturas de la ciudadanía. Lo anterior implica que el apoyo ciudadano a las instituciones es inherente en sociedades más democráticas. Pharr y Putnam (2000) refuerzan la idea de que esta asociación es unidireccional desde la democracia hacia la confianza, al sugerir que bajos niveles de confianza no necesariamente llevan a una postura negativa hacia los principios de la democracia. Torcal (2000) explora el caso de los países latinoamericanos para analizar cómo la democracia influye en la confianza institucional. El autor distingue en estos países características de una ciudadanía menos participativa con democracias recientes, tras experimentar regímenes de gobiernos autoritarios. De esta manera, la baja confianza y representación de las instituciones en los países latinoamericanos podría ser el resultado de democracias aún en desarrollo y un pasado inestable, que potencia una desafección a las nuevas democracias (Golder, 2005; Sarsfield y Echegaray, 2005). Finalmente, Montero, Torcal y Menezo (1998) destacan la incidencia de la legitimidad y satisfacción con las democracias en la desconfianza 
en las instituciones, lo cual se manifiesta a través de bajas tasas de participación electoral debido al desencanto, a la desilusión o a la decepción con la política.

Un segundo factor relevante que explica el proceso de confianza institucional se relaciona con el desempeño de las instituciones. La confianza no es entregada de forma natural; por el contrario, funciona desde parámetros de exigencia que deben ser cumplidos (Easton, 1965). De esta manera, el vínculo existente no es solo entre la confianza institucional y la democracia como tal, sino además con el desempeño democrático vigente. North (1990) plantea que la confianza interpuesta por la comunidad es una respuesta racional relacionada directamente con el accionar institucional. Así, se puede entender que esta confianza o desconfianza provendría de características internas de las instituciones, en particular de la capacidad de estas para realizar las tareas para las que fueron diseñadas. Por su parte, Norris (1999) observa que los países con niveles relativamente más altos de apoyo público son aquellos que tienen un mejor desempeño. Este efecto es mayor cuando el desempeño político se encuentra deteriorado o permanece bajo por un tiempo. Segovia et al. (2008) sostienen que los ciudadanos emiten sus juicios de confianza de manera estable a través de una evaluación que demuestre si una institución realiza las acciones que se espera de ella (capacidad) y las percepciones respecto de la voluntad o el interés de esta para actuar en pos del bienestar de la sociedad (benevolencia). Los autores encuentran que ambos factores son relevantes de manera individual para explicar los juicios de confianza, y esto genera un reforzamiento entre ellos.

Otro factor relevante que puede promover la confianza en las instituciones tiene que ver con la existencia y materialización de mecanismos de participación ciudadana. En este sentido, Putnam et al. (1993) aseveran que la asociación cívica y la participación social generan espacios de interacción social que promueven la comunicación y, por ende, el desarrollo de confianza por las "redes de compromiso cívico". Anderson y Tverdova (2001) plantean la existencia de una asociación entre la aprobación pública de las instituciones y la pertenencia a un bloque político, y sugieren que la confianza institucional podría reflejar un sesgo marcado por concepciones políticas. Sin embargo, se debe distinguir el apoyo político desde el apoyo o la confianza en las instituciones; mientras el primero se guía principalmente por cuestiones ideológicas, el apoyo a las instituciones se relaciona con elementos que garantizan representatividad ciudadana. Un estudio que se focaliza tanto en la participación ciudadana como en el factor anterior de desempeño es el realizado por Stoyan et al. (2016). La evidencia reportada por estos autores para República Dominicana y Haití avala el efecto positivo del actuar del Gobierno, medido en términos de desempeño económico, seguridad, calidad de los servicios y combate a la corrupción, sobre la confianza institucional en ambos países.

Adicionalmente, un factor clave que determina la confianza en las instituciones se relaciona con acciones de corrupción y aprovechamiento ilícito que se desencadenan en las instituciones. Bentham (1989) plantea que la voluntad actúa en cada ocasión bajo la presión de dos intereses opuestos y conflictivos: su participación fraccionaria en el interés universal y su propio interés particular y personal. Este incentivo es mucho más pronunciado en los funcionarios públicos (Echebarría, 2007). Las acciones de corrupción, colusión y conflicto de interés alcanzan una exposición mediática que promueve el interés de los medios, lo que facilita su divulgación e impacto en la confianza de la ciudadanía hacia las instituciones involucradas (Nye, Zelikow y King, 1997). Bowler y Karp (2004) agregan que aquellos personeros políticos involucrados en actos de corrupción no solo ven reducida su probabilidad de reelección, sino que además contribuyen a erosionar las bases de la confianza ciudadana hacia la institucionalidad política en democracia. Armah-Attoh, Gyimah-Boadi y Chikwanha (2007) estudian la incidencia de la corrupción en la aprobación de las instituciones en África. Sus resultados corroboran el argumento de que ciudadanías que perciben la corrupción como un fenómeno presente en el país tienen niveles de aprobación más bajos de sus instituciones y, por ende, de su confianza en estas. Morris y Klesner (2010) relevan el carácter endógeno de la relación 
entre corrupción y confianza institucional, puesto que el deterioro de esta última podría generar apatía o displicencia entre los ciudadanos respecto del actuar probo de los funcionarios del Estado y cierta tolerancia a las irregularidades reveladas a la luz pública. Los autores emplean el enfoque de variables instrumentales para evaluar el impacto de la experiencia, percepción y tolerancia a la corrupción sobre la confianza institucional en México, y encuentran evidencia estadística de un efecto negativo solo en las dos últimas.

Finalmente, la literatura identifica algunas hipótesis alternativas. Una de ellas indica que la confianza en las instituciones se correlaciona con el ciclo económico. Empleando datos de las encuestas Trust in Institutions and World Poll, elaboradas por Stevenson y Wolfers (2011) encuentran evidencia de que la confianza en los poderes del Estado, la banca y las instituciones financieras y los empresarios es procíclica, tanto para Estados Unidos como para el panel de países incluidos en el estudio. Es decir, un ciclo recesivo, sinónimo de mayores tasas de desempleo, reduciría los niveles de confianza dentro de la sociedad.

Una segunda hipótesis alternativa vincula el crimen con la confianza institucional. En un estudio realizado para 19 países de América Latina, Corbacho, Philipp y Ruiz-Vega (2015) remarcan que los niveles de criminalidad y victimización en la población reducen la confianza en los organismos de seguridad, lo cual debilita la cooperación, las instituciones y el ambiente económico para desarrollar negocios.

\section{Metodología}

Este estudio utiliza información de la encuesta AmericasBarometer, disponible gracias al LAPOP. Este proyecto se enmarca en una encuesta regular sobre las experiencias de los ciudadanos, sus evaluaciones y preferencias sobre cuestiones valóricas, gobierno, política, etc., en países del continente americano. Una de las características de esta encuesta es el esfuerzo para realizar comparaciones rigurosas y científicas en las Américas. Actualmente, la encuesta cubre 34 naciones, incluyendo Norte, Centro y Sur América, así como un significativo número de países del Caribe. Para el propósito de este estudio, se utiliza la ronda realizada en Chile en 2014, que permite evaluar un espectro amplio de instituciones ${ }^{1}$. En particular, el interés es explorar los factores clave que afectan la confianza institucional. La encuesta mencionada recoge información desde los ciudadanos que permite obtener percepciones sobre el nivel de confianza para un conjunto de instituciones enmarcadas en el plano judicial y de seguridad, político y legislativo, ejecutivo y de gobiernos locales e instituciones de carácter religioso. La pregunta específica es la siguiente: ¿hasta qué punto usted tiene confianza en...? Las respuestas se ordenan de manera categórica, donde el nivel mínimo 1 significa nada de confianza y 7 , mucha confianza. La tabla 1 muestra los porcentajes de respuestas para cada nivel de confianza en las instituciones de interés, tanto para Chile como para América Latina y el Caribe.

De acuerdo con la tabla 1, Chile muestra niveles más altos de confianza en las Fuerzas Armadas, Carabineros de Chile y el Ejecutivo, y un mayor porcentaje de respuestas en niveles bajos de confianza en los partidos políticos, la iglesia evangélica y el sistema judicial. En términos comparativos, las cifras sugieren que la población chilena confía más en la policía nacional, el poder ejecutivo y el Gobierno local en relación con sus pares de América Latina y el Caribe. Además, tal comparación indica que la desconfianza hacia el sistema judicial y la iglesia evangélica (o protestante) es mayor entre los chilenos.

Lo anterior no es un hecho concreto, sino que obedece a una tendencia creciente en los niveles de desconfianza institucional reportados en Chile durante los últimos diez años. Al respecto, la figura 1 da cuenta del porcentaje de la población chilena que exhibe los mayores niveles de desconfianza, definida esta como las respuestas categóricas de 1 a 3 a la pregunta: “¿hasta qué punto usted tiene confianza en...?". Tales cifras demuestran un evidente deterioro en la percepción que la ciudadanía tiene respecto de los poderes del estado chileno, en particular del poder legislativo.

1 La última ronda de AmericasBarometer aplicada en Chile fue en 2016; no obstante, esta excluye al sistema judicial y a organismos eclesiásticos en la medición de confianza institucional. 
Tabla 1. Niveles de confianza institucional en Chile y América Latina y el Caribe según tipo de institución, 2014

\begin{tabular}{|c|c|c|c|c|c|c|c|c|}
\hline & & \multicolumn{7}{|c|}{ Niveles de confianza (porcentaje) } \\
\hline & Instituciones & Nada & 2 & 3 & 4 & 5 & 6 & Mucho \\
\hline \multirow{9}{*}{ Chile } & Sistema judicial & $19,5 \%$ & $13,8 \%$ & $20,9 \%$ & $23,8 \%$ & $14,7 \%$ & $6,0 \%$ & $1,4 \%$ \\
\hline & Fuerzas armadas & $7,3 \%$ & $7,1 \%$ & $9,9 \%$ & $15,8 \%$ & $25,3 \%$ & $20,8 \%$ & $13,7 \%$ \\
\hline & Carabineros & $6,3 \%$ & $4,6 \%$ & $9,4 \%$ & $16,0 \%$ & $22,9 \%$ & $25,5 \%$ & $15,2 \%$ \\
\hline & Congreso Nacional & $17,5 \%$ & $12,9 \%$ & $22,3 \%$ & $23,7 \%$ & $16,0 \%$ & $5,9 \%$ & $1,6 \%$ \\
\hline & Partidos políticos & $30,2 \%$ & $15,3 \%$ & $19,2 \%$ & $18,8 \%$ & $11,5 \%$ & $4,2 \%$ & $0,8 \%$ \\
\hline & Poder ejecutivo & $8,5 \%$ & $5,7 \%$ & $8,2 \%$ & $18,9 \%$ & $25,8 \%$ & $20,5 \%$ & $12,6 \%$ \\
\hline & Municipalidad & $7,4 \%$ & $5,3 \%$ & $10,6 \%$ & $21,9 \%$ & $28,9 \%$ & $19,6 \%$ & $6,3 \%$ \\
\hline & Iglesia católica & $17,7 \%$ & $6,5 \%$ & $8,8 \%$ & $14,4 \%$ & $21,5 \%$ & $16,5 \%$ & $14,7 \%$ \\
\hline & Iglesia evangélica & $25,0 \%$ & $10,7 \%$ & $12,3 \%$ & $16,0 \%$ & $16,8 \%$ & $8,7 \%$ & $10,6 \%$ \\
\hline \multirow{9}{*}{$\begin{array}{c}\text { América Latina } \\
\text { y el Caribe } \\
\text { (excluido Chile) }\end{array}$} & Sistema judicial & $16,7 \%$ & $11,5 \%$ & $17,2 \%$ & $20,4 \%$ & $17,0 \%$ & $9,6 \%$ & $7,6 \%$ \\
\hline & Fuerzas armadas & $9,8 \%$ & $6,2 \%$ & $11,1 \%$ & $16,3 \%$ & $19,0 \%$ & $16,0 \%$ & $21,4 \%$ \\
\hline & Policía nacional & $17,7 \%$ & $10,7 \%$ & $15,5 \%$ & $18,0 \%$ & $16,8 \%$ & $10,9 \%$ & $10,5 \%$ \\
\hline & Congreso Nacional & $19,2 \%$ & $11,6 \%$ & $17,2 \%$ & $19,8 \%$ & $15,8 \%$ & $9,2 \%$ & $7,2 \%$ \\
\hline & Partidos políticos & $31,6 \%$ & $13,4 \%$ & $17,9 \%$ & $17,2 \%$ & $11,3 \%$ & $4,7 \%$ & $3,9 \%$ \\
\hline & Poder ejecutivo & $20,8 \%$ & $9,1 \%$ & $12,5 \%$ & $15,8 \%$ & $15,3 \%$ & $11,2 \%$ & $15,5 \%$ \\
\hline & Municipalidad & $17,0 \%$ & $10,2 \%$ & $16,4 \%$ & $19,8 \%$ & $16,7 \%$ & $10,3 \%$ & $9,6 \%$ \\
\hline & Iglesia católica & $17,0 \%$ & $6,0 \%$ & $8,4 \%$ & $11,4 \%$ & $13,2 \%$ & $12,7 \%$ & $31,2 \%$ \\
\hline & Iglesia evangélica & $25,3 \%$ & $8,9 \%$ & $11,2 \%$ & $12,2 \%$ & $12,1 \%$ & $9,3 \%$ & $21,1 \%$ \\
\hline
\end{tabular}

Nota: el cálculo de indicadores para América Latina y el Caribe utilizó una versión ampliada de la base de datos Grand Merge File de la Encuesta AmericasBarometer, incorporando la ronda 2014 para Ecuador, Bolivia, Venezuela y Argentina. Todos los indicadores fueron calculados utilizando el ponderador weighT1500.

Fuente: elaboración propia, a partir de datos de la encuesta AmericasBarometer 2014.

En efecto, de acuerdo con la medición de 2016, un $78,9 \%$ y un $61 \%$ de la población desconfía de los partidos políticos y del Congreso Nacional, respectivamente; cifras que representan un incremento superior al $60 \%$ respecto de lo que se reportó para 2006. Adicionalmente, el sistema judicial y la iglesia evangélica son instituciones cuyos niveles de desconfianza bordean el $50 \%$ en la medición de 2014, la que no superaba el $40 \%$ en 2010. Por el contrario, las Fuerzas Armadas, los Carabineros de Chile y el Gobierno local (i. e., municipios) son instituciones que registran los menores índices durante el periodo 2006-2016, a pesar de la creciente tendencia de desconfianza en Chile.

La literatura sobre confianza institucional en América Latina y el Caribe exhibe cierta diversidad en la estrategia empírica seleccionada para el análisis. Al respecto, Morris y Klesner (2010) aplican mínimos cuadrados en tres etapas para determinar la relación entre la percepción de corrupción y confianza institucional en México, empleando la ronda 2004 de la encuesta AmericasBarometer. Stoyan et al. (2016), quienes también explotan la encuesta LAPOP, realizan una estimación por mínimos cuadrados para el grado de confianza en el Gobierno, en la cual esta variable dependiente es construida a partir de una escala aditiva que involucra solo aquellas preguntas vinculadas al grado de confianza en el Servicio Electoral, en el Congreso Nacional, en la Corte Suprema y tribunales de justicia de Haití y República Dominicana. Segovia et al. (2008) emplean el análisis de regresión jerárquico (o análisis multinivel) para capturar el efecto de la benevolencia y la capacidad para ejercer funciones sobre los niveles de confianza de un conjunto de instituciones chilenas.

A diferencia de esos estudios, la presente investigación estima un modelo probit ordenado que se ajusta mejor cuando la variable dependiente es de naturaleza categórica ordenada, como este caso donde la confianza institucional se mide en una escala de 1 a 7 . 


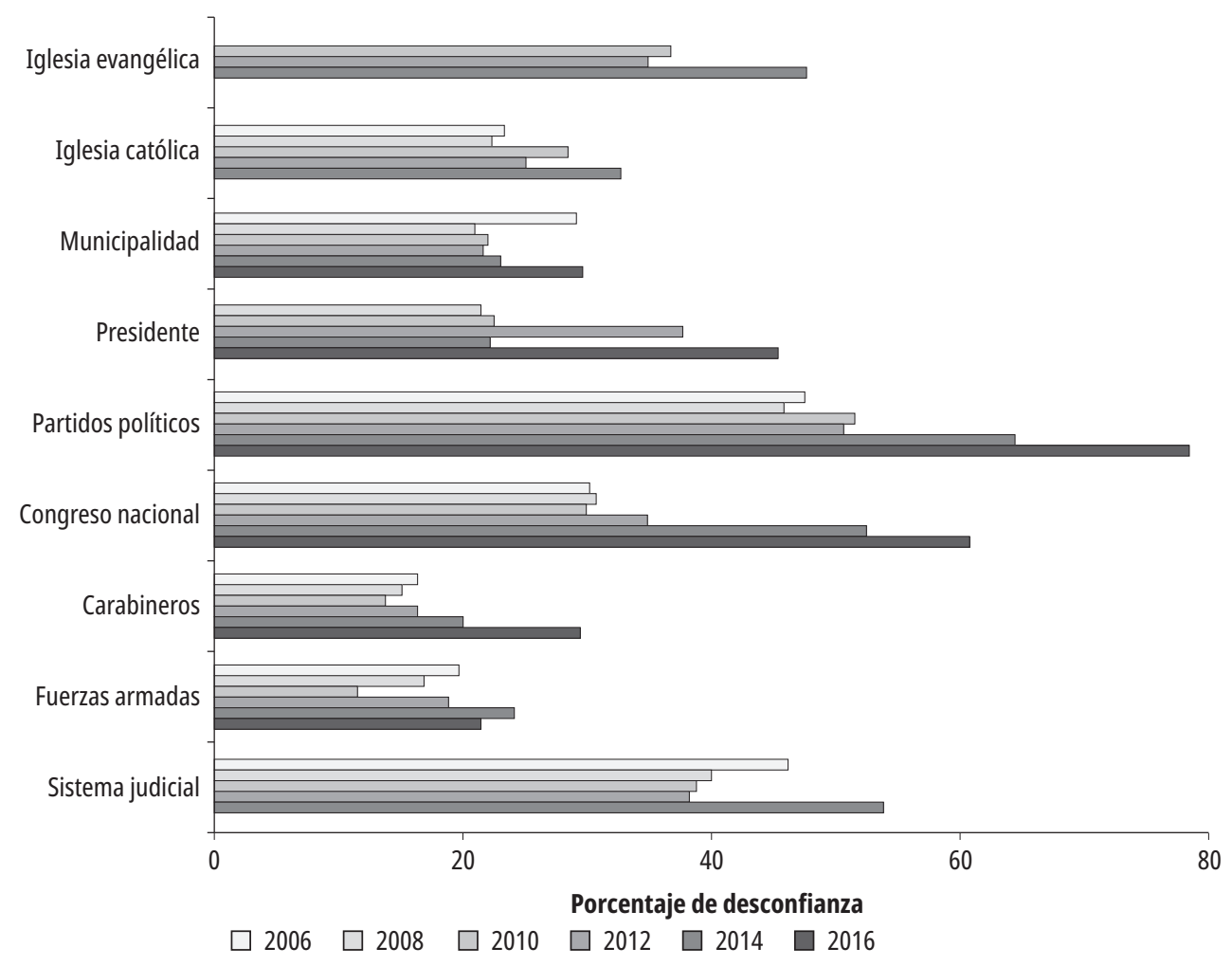

Figura 1. Porcentaje de desconfianza institucional en Chile según tipo de institución, periodo 2006-2016 Fuente: elaboración propia, a partir de análisis de Encuesta AmericasBarometer.

Dado esto, sea una variable latente no observada para la confianza institucional definida como $C I_{i}^{*}$, el modelo por estimar es el siguiente:

$$
C I_{i}^{*}=x \beta+\varepsilon_{i}
$$

Donde $\varepsilon \sim N(0,1)$

La variable latente no es observada; no obstante, vemos en los datos elecciones sobre los niveles de confianza de acuerdo con lo siguiente:

$$
\begin{gathered}
C I=1 s i C I_{i}^{*} \leq \propto_{1} \\
C I=2 s i \propto_{1}<C I_{i}^{*} \leq \propto_{2} \\
C I=3 s i \propto_{2} \leq C I_{i}^{*} \leq \propto_{3} \\
C I=4 s i \propto_{3} \leq C I_{i}^{*} \leq \propto_{4} \\
C I=5 s i \propto_{4} \leq C I_{i}^{*} \leq \propto_{5} \\
C I=6 s i \propto_{5} \leq C I_{i}^{*} \leq \propto_{6} \\
C I=7 s i \propto_{6}<C I_{i}^{*}
\end{gathered}
$$

Donde $\propto_{1} \ldots \propto_{6}$ son los puntos de corte o parámetros umbrales. El modelo es estimado por el método de máxima verosimilitud.

Siguiendo la revisión de la literatura para explicar la confianza institucional, se incluyen variables explicativas que capturan diferencias socioeconómicas entre los encuestados, de percepción sobre la democracia, el desempeño institucional, la participación de la sociedad civil y la corrupción. En primer lugar, se incorporan una serie de características socioeconómicas y de localización de los encuestados. De esta manera, se considera EDU el nivel de educación medido en años de estudio; EDAD los años de vida del encuestado; $O C P$ una variable dicotómica que toma el valor de 1 si la persona se encuentra ocupada y 0 en otro caso; $E C V$ el estado civil de la persona tomando el valor de 1 si es casado y 0 otros caso; MEST INDG son definidas como variables dummies identificando la etnia del encuestado como mestizo o indígena, respectivamente, tomando 
el valor de 1 si pertenece a alguna de las etnias anteriores y 0 en otro caso; RELG hace referencia a si la persona es miembro de una organización religiosa, tomando el valor de 1 si es miembro y 0 en caso contrario; URB es una variable dummy que toma el valor de 1 si la persona vive en una zona urbana y 0 en una zona rural; NOR y CENT son variables dummies por zona, y queda como base la zona sur; TMM es una variable que mide el tamaño de un municipio siguiendo tres categorías: municipios grandes que contienen más de 100.000 habitantes (3), medianos que tienen 25.000 y 100.000 habitantes (2) y pequeños con menos de 25.000 habitantes (1).

En relación con las variables que capturan diferencias en percepción sobre la democracia, se incorpora RSP.IP, que mide el grado de respeto hacia las instituciones políticas en una escala de 1 a 7; C.ELE mide la confianza en las elecciones en un rango de 1 a 7; DEMCR y AUTOR son variables dummies que recogen preferencias por un Gobierno democrático o un Gobierno autoritario, respectivamente, considerando como base los indiferentes.

Para el caso de las variables asociadas al desempeño institucional, se incluye SEGUR que se relaciona con la percepción de la seguridad del barrio, tomando valores en una escala de 1 a 4 , donde 1 es muy inseguro y 4 muy seguro; CAST.J denota la confianza respecto a que el sistema judicial castigue al culpable, tomando valores entre 1 y 4 , donde el valor mínimo indica nada de confianza y el valor más alto mucha confianza; la aprobación del presidente de turno denotada por la variable AP.PRES en una escala de 1 a 5 ; JUIJUS es una variable que identifica si el encuestado considera que los tribunales de justicia garantizan un juicio justo en una escala de 1 a 7; DERBA es una variable que informa si el encuestado percibe si los derechos básicos están protegidos en una escala de 1 a 7; DERHU es la variable que denota si los derechos humanos son respetados por las Fuerzas Armadas, y se mide en una escala de 1 a 7; MEDIC mide la confianza en el servicio médico público en una escala de 1 a 7 ; PENPUB responde a la percepción de la ciudadanía con respecto a si el Gobierno está interesado en el pensamiento de la comunidad en una escala de 1 a 7; SEGCIU es la variable que mide el desempeño del Gobierno en materia de seguridad ciudadana en una escala de 1 a 7 ; DESPOL $L$ hace lo mismo con el desempeño de las policías en los barrios; TIMPOL mide, de forma alternativa, los tiempos de demora de la policía en una escala de 1 a 6 , donde 1 indica menos demora y 6 más tiempo de demora; EDUC mide la percepción sobre la calidad de las escuelas públicas en una escala de 1 a 4, y ECON captura la percepción de la ciudadanía sobre la situación económica del país con respecto al año anterior, con respuestas que se categorizan del 1 al 3, donde 1 indica peor, 2 igual y 3 mejor.

Para el caso de la participación, se incorporan variables que expresan un mayor o menor involucramiento de la comunidad con las instituciones de interés. REUPOL se define como una variable que captura el grado de participación en reuniones de movimientos políticos, y está estructurada por recurrencia a estos en cuatro categorías ordenadas de menor a mayor, partiendo desde nunca (1), pasando por una o dos veces al año (2), una o dos veces al mes (3) y una vez por semana (4); REUREL, de manera análoga, considera la asistencia a reuniones en instituciones religiosas; REUMUN es una variable dummy que toma el valor de 1 si la persona asistió a una sesión municipal o cabildo abierto en los últimos doce meses y 0 en otro caso, y SIMPOL se describe como una variable dummy, tomando el valor de 1 si el encuestado simpatiza con algún partido político y 0 en otro caso.

Finalmente, para la dimensión corrupción se incluye: DESCOR que hace alusión a si el Gobierno muestra una actitud de combate contra la corrupción en una escala de 1 a 7; SOBOR es una variable dummy que toma el valor de 1 si la persona alguna vez ha recibido una solicitud de soborno en algunas de las instituciones de interés.

Para el caso particular de las instituciones religiosas, se agrega $A B O R T$, que toma el valor de $1 \mathrm{si}$ la persona justifica el aborto en el caso de que exista riesgo vital para la madre, e $I G U A L$, que toma el valor de 1 si la persona está de acuerdo con el 
matrimonio entre parejas del mismo sexo en una escala de 1 a 10. La tabla 2 presenta las estadísticas descriptivas para las variables explicativas del modelo de confianza institucional.

Tabla 2. Estadística descriptiva de las variables explicativas

\begin{tabular}{|c|c|c|c|c|c|}
\hline Variables & Observaciones & Media & Desv. estándar & Mínimo & Máximo \\
\hline \multicolumn{6}{|c|}{ Socioeconómicas } \\
\hline EDU & 1565 & 10,491 & 4,054 & 0 & 17 \\
\hline EDAD & 1571 & 49,386 & 17,442 & 18 & 99 \\
\hline OCP & 1571 & 0,423 & 0,494 & 0 & 1 \\
\hline ECV & 1571 & 0,473 & 0,499 & 0 & 1 \\
\hline RELG & 1457 & 0,887 & 0,316 & 0 & 1 \\
\hline MEST & 1547 & 0,462 & 0,499 & 0 & 1 \\
\hline INDG & 1547 & 0,045 & 0,206 & 0 & 1 \\
\hline OTRO & 1547 & 0,494 & 0,500 & 0 & 1 \\
\hline URB & 1571 & 0,867 & 0,340 & 0 & 1 \\
\hline NOR & 1571 & 0,118 & 0,323 & 0 & 1 \\
\hline CENT & 1571 & 0,626 & 0,484 & 0 & 1 \\
\hline SUR & 1571 & 0,255 & 0,436 & 0 & 1 \\
\hline TMM & 1571 & 2,283 & 0,774 & 1 & 3 \\
\hline \multicolumn{6}{|c|}{ Democracia } \\
\hline RSP.IP & 1523 & 4,566 & 1,675 & 1 & 7 \\
\hline DEMCR & 1404 & 0,733 & 0,443 & 0 & 1 \\
\hline AUTOR & 1404 & 0,150 & 0,357 & 0 & 1 \\
\hline INDF & 1404 & 0,118 & 0,322 & 0 & 1 \\
\hline C. ELE & 1518 & 4,816 & 1,542 & 1 & 7 \\
\hline \multicolumn{6}{|c|}{ Desempeño } \\
\hline SEGUR & 1565 & 2,718 & 0,876 & 1 & 4 \\
\hline CAST.J & 1546 & 1,904 & 0,889 & 1 & 4 \\
\hline jUIJUS & 1523 & 3,332 & 1,592 & 1 & 7 \\
\hline DERBA & 1511 & 3,963 & 1,473 & 1 & 7 \\
\hline DERHU & 1444 & 4,657 & 1,515 & 1 & 7 \\
\hline PENPUB & 1514 & 3,411 & 1,745 & 1 & 7 \\
\hline TIMPOL & 1544 & 2,837 & 1,298 & 1 & 6 \\
\hline DEPOL & 1539 & 2,591 & 0,749 & 1 & 4 \\
\hline SEGCIU & 1435 & 4,004 & 1,448 & 1 & 7 \\
\hline MEDIC & 1544 & 4,076 & 1,694 & 1 & 7 \\
\hline EDUC & 1220 & 2,442 & 0,726 & 1 & 4 \\
\hline ECON & 1542 & 2,011 & 0,619 & 1 & 3 \\
\hline AP. PRES & 1420 & 3,478 & 0,830 & 1 & 5 \\
\hline
\end{tabular}


Continúa Tabla 2. Estadística descriptiva de las variables explicativas

\begin{tabular}{|c|c|c|c|c|c|}
\hline Variables & Observaciones & Media & Desv. estándar & Mínimo & Máximo \\
\hline \multicolumn{6}{|c|}{ Participación } \\
\hline REUPOL & 1566 & 1,047 & 0,305 & 1 & 4 \\
\hline REUREL & 1569 & 1,811 & 1,129 & 1 & 4 \\
\hline REUMUN & 1566 & 0,067 & 0,250 & 0 & 1 \\
\hline SIMPOL & 1497 & 0,131 & 0,337 & 0 & 1 \\
\hline \multicolumn{6}{|c|}{ Corrupción } \\
\hline DESCORR & 1369 & 3,807 & 1,473 & 1 & 7 \\
\hline SOBOR & 1571 & 0,069 & 0,253 & 0 & 1 \\
\hline \multicolumn{6}{|c|}{ Valórico } \\
\hline IGUAL & 1507 & 5,020 & 3,586 & 1 & 10 \\
\hline ABOR & 1435 & 0,657 & 0,475 & 0 & 1 \\
\hline
\end{tabular}

Fuente: elaboración propia.

\section{Resultados}

En esta sección se discuten los resultados de las estimaciones para cada grupo de instituciones. La tabla 3 muestra los parámetros estimados para los modelos y explica la confianza en instituciones judiciales y de seguridad, particularmente en el sistema judicial, las Fuerzas Armadas y los Carabineros de Chile.

Tabla 3. Estimaciones para la confianza en instituciones judiciales y seguridad

\begin{tabular}{|c|c|c|c|}
\hline Variables & Sistema judicial & Fuerzas Armadas & Carabineros de Chile \\
\hline \multicolumn{4}{|c|}{ Socioeconómico } \\
\hline \multirow{2}{*}{ EDU } & $0,024^{* *}$ & $-0,005$ & $-0,004$ \\
\hline & $(0,010)$ & $(0,010)$ & $(0,010)$ \\
\hline \multirow{2}{*}{ EDAD } & 0,001 & 0,003 & $0,006^{* *}$ \\
\hline & $(0,002)$ & $(0,002)$ & $(0,002)$ \\
\hline \multirow{2}{*}{ OCP } & $-0,129^{*}$ & $-0,070$ & 0,072 \\
\hline & $(0,071)$ & $(0,068)$ & $(0,069)$ \\
\hline \multirow{2}{*}{ ECV } & $-0,190^{* * *}$ & $-0,030$ & 0,064 \\
\hline & $(0,069)$ & $(0,069)$ & $(0,069)$ \\
\hline \multirow{2}{*}{ MEST } & 0,114 & $-0,041$ & $-0,043$ \\
\hline & $(0,073)$ & $(0,071)$ & $(0,072)$ \\
\hline \multirow{2}{*}{ INDG } & $-0,045$ & $-0,273^{*}$ & $-0,059$ \\
\hline & $(0,164)$ & $(0,159)$ & $(0,157)$ \\
\hline \multirow{2}{*}{ URB } & $-0,151$ & $0,217^{* *}$ & 0,088 \\
\hline & $(0,102)$ & $(0,096)$ & $(0,105)$ \\
\hline \multirow{2}{*}{ NOR } & $-0,438 * * *$ & $-0,254^{* *}$ & $-0,411^{* * *}$ \\
\hline & $(0,114)$ & $(0,114)$ & $(0,108)$ \\
\hline
\end{tabular}


Continuación Tabla 3. Estimaciones para la confianza en instituciones judiciales y seguridad

\begin{tabular}{|c|c|c|c|}
\hline Variables & Sistema judicial & Fuerzas Armadas & Carabineros de Chile \\
\hline \multirow{2}{*}{ CENT } & $-0,202^{* * *}$ & $-0,137^{*}$ & $-0,023$ \\
\hline & $(0,076)$ & $(0,081)$ & $(0,084)$ \\
\hline \multirow{2}{*}{ TMM } & $-0,069$ & 0,030 & $0,139^{* * *}$ \\
\hline & $(0,049)$ & $(0,047)$ & $(0,049)$ \\
\hline \multicolumn{4}{|c|}{ Democracia } \\
\hline \multirow{2}{*}{ RSP.IP } & 0,037 & $0,098 * * *$ & $0,105^{* * *}$ \\
\hline & $(0,027)$ & $(0,029)$ & $(0,026)$ \\
\hline \multirow{2}{*}{ DEMCR } & $-0,066$ & 0,012 & 0,001 \\
\hline & $(0,117)$ & $(0,111)$ & $(0,115)$ \\
\hline \multirow{2}{*}{ AUTOR } & 0,032 & $0,280^{* *}$ & $-0,054$ \\
\hline & $(0,132)$ & $(0,130)$ & $(0,134)$ \\
\hline \multicolumn{4}{|c|}{ Desempeño } \\
\hline \multirow{2}{*}{ SEGUR } & 0,027 & 0,074 & $0,107^{* *}$ \\
\hline & $(0,050)$ & $(0,046)$ & $(0,047)$ \\
\hline \multirow{2}{*}{ CAST.J } & $0,290^{* * *}$ & $-0,015$ & 0,028 \\
\hline & $(0,045)$ & $(0,042)$ & $(0,042)$ \\
\hline \multirow{2}{*}{ JUIJUS } & $0,353^{* * *}$ & 0,027 & 0,026 \\
\hline & $(0,030)$ & $(0,026)$ & $(0,025)$ \\
\hline \multirow{2}{*}{ DERBA } & $0,124^{* * *}$ & $0,083^{* *}$ & $0,078^{* *}$ \\
\hline & $(0,034)$ & $(0,034)$ & $(0,032)$ \\
\hline \multirow{2}{*}{ DERHU } & 0,041 & $0,401^{* * *}$ & $0,271^{* \star *}$ \\
\hline & $(0,029)$ & $(0,035)$ & $(0,031)$ \\
\hline \multirow{2}{*}{ PENPUB } & $0,062^{* * *}$ & 0,013 & $-0,008$ \\
\hline & $(0,024)$ & $(0,024)$ & $(0,023)$ \\
\hline \multirow{2}{*}{ TIMPOL } & $-0,017$ & 0,016 & $-0,106^{* * *}$ \\
\hline & $(0,034)$ & $(0,032)$ & $(0,031)$ \\
\hline \multirow{2}{*}{ DESPOL } & $-0,070$ & 0,062 & $0,337^{* \star *}$ \\
\hline & $(0,059)$ & $(0,055)$ & $(0,058)$ \\
\hline \multirow{2}{*}{ SEGCIU } & $0,067^{*}$ & $0,082^{* *}$ & $0,151^{* * *}$ \\
\hline & $(0,040)$ & $(0,040)$ & $(0,037)$ \\
\hline \multicolumn{4}{|c|}{ Participación } \\
\hline \multirow{2}{*}{ REUPOL } & $-0,0553$ & $-0,0351$ & 0,0332 \\
\hline & $(0,119)$ & $(0,137)$ & $(0,143)$ \\
\hline \multirow{2}{*}{ SIMPOL } & 0,100 & $-0,127$ & $-0,178^{*}$ \\
\hline & $(0,0896)$ & $(0,0980)$ & $(0,101)$ \\
\hline \multicolumn{4}{|c|}{ Corrupción } \\
\hline \multirow{2}{*}{ DESCORR } & $0,130^{\star * *}$ & $-0,087^{\star *}$ & $-0,031$ \\
\hline & $(0,037)$ & $(0,037)$ & $(0,034)$ \\
\hline \multirow{2}{*}{ SOBOR } & 0,218 & $0,377^{* * *}$ & $-0,029$ \\
\hline & $(0,142)$ & $(0,143)$ & $(0,113)$ \\
\hline
\end{tabular}


Continuación Tabla 3. Estimaciones para la confianza en instituciones judiciales y seguridad

\begin{tabular}{l|cc|c} 
Variables & Sistema judicial & Fuerzas Armadas & Carabineros de Chile \\
\multirow{2}{*}{ Constant cuT1 } & $1,803^{* * *}$ & $1,401^{* * *}$ & $1,885^{* * *}$ \\
\cline { 2 - 4 } Constant cuT2 & $(0,367)$ & $(0,355)$ & $(0,341)$ \\
\hline & $2,441^{* * *}$ & $1,860^{* * *}$ & $2,362^{* * *}$ \\
\hline \multirow{2}{*}{ Constant cut3 } & $(0,371)$ & $(0,358)$ & $(0,344)$ \\
\hline \multirow{2}{*}{ Constant cut4 } & $3,205^{* * *}$ & $2,299^{* * *}$ & $2,911^{* * *}$ \\
\hline \multirow{2}{*}{ Constant cut5 } & $(0,376)$ & $(0,359)$ & $(0,346)$ \\
\hline \multirow{2}{*}{ Constant cut6 } & $4,090^{* * *}$ & $2,912^{* * *}$ & $3,540^{* * *}$ \\
\hline & $(0,380)$ & $(0,363)$ & $(0,351)$ \\
\hline Observaciones & $5,066^{* * *}$ & $3,732^{* * *}$ & $4,367^{* * *}$ \\
\hline
\end{tabular}

Nota: *** estadísticamente significativos al $99 \%$ de confianza; **estadísticamente significativos al $95 \%$ de confianza; *estadísticamente significativos al $90 \%$ de confianza. Errores estándar robustos en paréntesis.

Fuente: elaboración propia.

Los resultados muestran que la confianza institucional en las Fuerzas Armadas depende de la zona de residencia. Mientras los individuos que residen en la zona centro y norte del país depositan menos confianza en la institución que en la zona sur, los habitantes de zonas urbanas tienden a confiar más en las Fuerzas Armadas; por su parte, las personas que manifiestan pertenecer a alguna etnia indígena confían menos. Lo anterior puede ser resultado de una serie de eventos ocurridos en el sur del país desde hace décadas, que han dado origen a un conflicto del Estado con el pueblo originario mapuche. Este resultado es preocupante porque una sociedad fragmentada étnica o socialmente presenta menores niveles de cohesión social, lo que pone en riesgo la implementación de una política económica con enfoque de largo plazo, encauzada a la resolución de conflictos sociales (Easterly et al., 2006).

En relación con los aspectos democráticos, se tiene que las personas que manifiestan un acuerdo con la afirmación "en algunas circunstancias un Gobierno autoritario puede ser preferible" tienen una incidencia positiva y significativa en el nivel de confianza en las Fuerzas Armadas. Este resultado podría reflejar la persistencia de un legado antidemocrático en parte de la población chilena, como consecuencia de un régimen autoritario que duró 17 años. Por último, los resultados sugieren que las personas que piensan que las Fuerzas Armadas no respetan los derechos humanos confían menos en la institución. Este último resultado también podría responder al legado de una dictadura militar con probadas violaciones a los derechos humanos en Chile.

Respecto a la confianza en la policía o Carabineros, se observa que las personas que residen en la zona norte y centro confían menos que las de la zona sur del país, y que los individuos que residen en localidades de mayor tamaño tienen más confianza en la policía. Asimismo, se encuentra que los habitantes que pertenecen a algún tipo de religión y de mayor edad confían más en la policía.

Esto último podría ser explicado porque con mayor frecuencia son los individuos de rangos etarios más jóvenes los que se ven envueltos en problemas policiales. En cuanto a las variables que miden del desempeño de las policías, se encuentra que si los individuos evalúan de manera positiva el desempeño de la institución, estos tienden a confiar más en esta. Además, los individuos que manifiestan mayor seguridad en su barrio ante situaciones 
de robo y delincuencia presentan un mayor nivel de confianza institucional. Este resultado es similar a lo reportado por Corbacho et al. (2015) para América Latina, cuya evidencia confirma una relación negativa entre el grado de victimización criminal de la población y la confianza hacia la policía. De manera análoga, se encuentra que en la medida que aumentan los tiempos de respuesta de la policía, la ciudadanía baja su nivel de confianza en la institución. Finalmente, la percepción sobre el respeto a los derechos humanos por parte de las Fuerzas Armadas también es un factor que afecta la confianza en la policía. En Chile, ambas instituciones jugaron un rol clave en el régimen autoritario en Chile, con violaciones a los derechos humanos que también involucraron a la policía

En lo que tiene que ver con el sistema judicial, existe una relación significativa y positiva entre variables de desempeño y confianza institucional. En particular, las personas que tienen mayor confianza en que el sistema judicial castigue a los culpables y las que creen que las cortes realizan un juicio justo tienden a niveles más altos de confianza en estas instituciones.

De igual forma, la confianza en el sistema judicial es mayor, en la medida que las personas creen que los derechos básicos están protegidos. Además, las personas que habitan las zonas norte y centro del país confían más en el Sistema judicial, así como los habitantes de poblados más grandes. A esto se suma el hecho de que si las personas creen que ha habido un buen desempeño del Gobierno en cuanto a la lucha contra la corrupción confían más en el sistema judicial. Este resultado es similar al reportado por Stoyan et al. (2016) para República Dominicana y Haití durante el periodo 2008-2012, donde el desempeño del Gobierno respecto del combate a la corrupción incrementa la confianza institucional; lo contrario ocurre para el caso de las Fuerzas Armadas. Por último, los resultados sugieren que los individuos con mayor nivel de educación formal confían más en el sistema judicial, resultado opuesto al que encontraron Morris y Klesner (2010) para México, empleando la ronda 2004 de la encuesta LAPOP.

La tabla 4 presenta los parámetros estimados para el modelo y explica la confianza institucional en los partidos políticos y en el Congreso Nacional.
Tabla 4. Estimaciones para la confianza en instituciones políticas y legislativas

\begin{tabular}{|c|c|c|}
\hline Variables & Partidos políticos & Congreso Nacional \\
\hline \multicolumn{3}{|c|}{ Socioeconómicas } \\
\hline \multirow{2}{*}{ EDU } & 0,014 & 0,014 \\
\hline & $(0,009)$ & $(0,009)$ \\
\hline \multirow{2}{*}{ EDAD } & 0,002 & 0,002 \\
\hline & $(0,002)$ & $(0,002)$ \\
\hline \multirow{2}{*}{ ECV } & $-0,0911$ & $-0,133^{* *}$ \\
\hline & $(0,066)$ & $(0,065)$ \\
\hline \multirow{2}{*}{ MEST } & $0,159 * *$ & 0,102 \\
\hline & $(0,067)$ & $(0,068)$ \\
\hline \multirow{2}{*}{ INDG } & 0,290 & $-0,093$ \\
\hline & $(0,177)$ & $(0,168)$ \\
\hline \multirow{2}{*}{ RELG } & $0,438^{* * *}$ & 0,280 ** \\
\hline & $(0,107)$ & $(0,111)$ \\
\hline \multirow{2}{*}{ URB } & $-0,302^{* * *}$ & $-0,030$ \\
\hline & $(0,105)$ & $(0,100)$ \\
\hline \multirow{2}{*}{ NOR } & $-0,100$ & $-0,069$ \\
\hline & $(0,118)$ & $(0,106)$ \\
\hline \multirow{2}{*}{ CENT } & 0,082 & $-0,002$ \\
\hline & $(0,077)$ & $(0,076)$ \\
\hline \multirow{2}{*}{ TMM } & $-0,067$ & $-0,057$ \\
\hline & $(0,046)$ & $(0,046)$ \\
\hline \multicolumn{3}{|c|}{ Democracia } \\
\hline \multirow{2}{*}{ C.ELE } & $0,082^{* * *}$ & $0,104^{* * *}$ \\
\hline & $(0,026)$ & $(0,028)$ \\
\hline \multirow{2}{*}{ RSP.IP } & $0,070^{* * *}$ & $0,081^{* * *}$ \\
\hline & $(0,025)$ & $(0,026)$ \\
\hline \multicolumn{3}{|c|}{ Desempeño } \\
\hline \multirow{2}{*}{ DERBA } & $0,142^{* * *}$ & $0,220 * * *$ \\
\hline & $(0,030)$ & $(0,033)$ \\
\hline \multirow{2}{*}{ PENPUB } & $0,086^{* * *}$ & $0,066^{* * *}$ \\
\hline & $(0,022)$ & $(0,022)$ \\
\hline \multicolumn{3}{|c|}{ Participación } \\
\hline \multirow{2}{*}{ REUPOL } & $0,284^{* * *}$ & 0,104 \\
\hline & $(0,101)$ & $(0,082)$ \\
\hline \multirow{2}{*}{ SIMPOL } & $0,363^{* * *}$ & 0,076 \\
\hline & $(0,086)$ & $(0,089)$ \\
\hline \multicolumn{3}{|c|}{ Corrupción } \\
\hline \multirow{2}{*}{ DESCORR } & $0,139 * * *$ & $0,126 * * *$ \\
\hline & $(0,028)$ & $(0,028)$ \\
\hline
\end{tabular}




\begin{tabular}{|c|c|c|}
\hline Variables & Partidos políticos & Congreso Nacional \\
\hline \multirow{2}{*}{ SOBOR } & $-0,129$ & $-0,027$ \\
\hline & $(0,135)$ & $(0,123)$ \\
\hline \multicolumn{3}{|c|}{ Umbrales } \\
\hline \multirow{2}{*}{ Constant cuT1 } & $2,083^{* * *}$ & $1,642^{* * *}$ \\
\hline & $(0,263)$ & $(0,244)$ \\
\hline \multirow{2}{*}{ Constant cuT2 } & $2,546^{* \star *}$ & $2,173^{\star * *}$ \\
\hline & $(0,264)$ & $(0,246)$ \\
\hline \multirow{2}{*}{ Constant cut3 } & $3,134^{* * *}$ & $2,844^{* * *}$ \\
\hline & $(0,268)$ & $(0,252)$ \\
\hline \multirow{2}{*}{ Constant cut4 } & $3,812^{* * *}$ & $3,640^{* * *}$ \\
\hline & $(0,271)$ & $(0,259)$ \\
\hline \multirow{2}{*}{ Constant cut5 } & $4,641^{* * *}$ & $4,499 * * *$ \\
\hline & $(0,283)$ & $(0,270)$ \\
\hline \multirow{2}{*}{ Constant cut6 } & $5,546^{* * *}$ & $5,325^{\star * *}$ \\
\hline & $(0,318)$ & $(0,292)$ \\
\hline Observaciones & 1,114 & 1,108 \\
\hline
\end{tabular}

Nota: *** estadísticamente significativos al $99 \%$ de confianza; **estadísticamente significativos al $95 \%$ de confianza; *estadísticamente significativos al $90 \%$ de confianza. Errores estándar robustos en paréntesis.

Fuente: elaboración propia

En relación con las variables socioeconómicas y de localización, las personas casadas confían menos en el Congreso Nacional que las solteras. Este fenómeno podría obedecer al hecho de que las personas que han constituido una familia podrían tener una visión más crítica de la política, lo cual posiblemente está relacionado con un mayor interés en la contingencia del país. Además, los resultados muestran que las personas que reportan como etnia "mestizo" tienden a confiar más en los partidos políticos. La religiosidad también aumenta la confianza en los partidos políticos y en el Congreso, resultado alineado con la evidencia encontrada por Stoyan et al. (2016) para República Dominicana en 2008 y 2010, donde una mayor asistencia al culto religioso incrementa la confianza institucional en ese país caribeño. Se argumenta que las personas religiosas confiarían en las instituciones de la misma forma como creen en Dios, considerando a las instituciones un ente representante de este. Los resultados muestran que las personas que residen en zonas urbanas confían menos en los partidos políticos, lo que puede explicarse por la existencia de una población más crítica, pero no necesariamente más instruida, en las zonas urbanas en relación con las zonas rurales. Esto se evidencia en las movilizaciones sociales y actos masivos que demuestran el descontento público, situación que no se da con la misma efervescencia ni representatividad en zonas rurales.

Referente a los aspectos democráticos, cuanto mayor sea la confianza en las elecciones dentro de un sistema democrático, mayor será la confianza en los partidos políticos y en el Congreso Nacional. Un resultado similar se encuentra cuando los individuos muestran un mayor respeto por las instituciones. Este resultado es respaldado por Montero et al. (1998), quienes destacan la incidencia de la legitimidad y satisfacción con las democracias en la desconfianza en las instituciones.

Para capturar la incidencia del desempeño institucional en la confianza, se introduce la variable que recaba percepciones respecto a si los derechos básicos están protegidos, así como percepciones relativas a si el Gobierno está interesado en lo que piensa la gente. Los resultados muestran un efecto positivo y significativo de ambas variables, y evidencian una relación entre la confianza institucional y la manera como los intereses de la ciudadanía son incorporados en el quehacer político.

En el tema de las variables de participación, se tiene un efecto positivo de la asistencia a reuniones de movimientos políticos en la confianza en los partidos políticos. Una participación activa de la ciudadanía en las instituciones de interés fortalece las relaciones de confianza, lo cual la hace partícipe en las decisiones y crea un compromiso con estas. Asimismo, los resultados muestran que las personas con una mayor simpatía hacia los partidos políticos suelen tener más confianza en estos; este aspecto podría responder a cuestiones ideológicas que refuerzan la confianza en ese tipo de instituciones. Anderson y Tverdova (2001) corroboran este resultado al argumentar la existencia de un vínculo entre la aprobación pública de las instituciones y la participación política, sugiriendo que la confianza institucional podría ser influenciada por cuestiones ideológicas y políticas.

Finalmente, en relación con las variables de corrupción, si la ciudadanía percibe que el Gobierno 
ha hecho esfuerzos importantes para combatir la corrupción, los niveles de confianza en los partidos políticos y el Congreso Nacional aumentan. De esta manera, una mala gestión del Gobierno en términos de corrupción podría aumentar substancialmente la insatisfacción de las personas y su grado de desconfianza institucional. En este sentido, la evidencia internacional arroja resultados similares a los reportados. Bowler y Karp (2004) afirman que si el electorado se entera de políticos envueltos en escándalos de corrupción, aumentaría la desaprobación del Congreso de Estados Unidos y se reduciría la confianza en el Parlamento británico.

En la tabla 5 se presentan los resultados para las instituciones de gobernanza nacional y local, en particular lo referente a la confianza en el Ejecutivo y las municipalidades.

En lo concerniente a las variables socioeconómicas, las personas ocupadas confían menos tanto en el ejecutivo como en las municipalidades. De manera similar al resto de las instituciones, la confianza institucional es menor en la zona norte y centro del país en relación con la zona sur, así como en las ciudades más grandes.

En cuanto a las variables de democracia, los resultados señalan que las personas que evalúan de mejor forma el proceso eleccionario tienen una mayor confianza en el ejecutivo y las municipalidades. Esta mejor evaluación del proceso eleccionario puede ser fruto del grado de autonomía formal del Servicio Electoral de Chile y del diseño institucional implementado tras la transición democrática, situación alineada con la hipótesis de confianza institucional y la evidencia registrada por Rosas (2010) para América Latina.

Con respecto al desempeño, una alta aprobación del presidente de turno parece ser clave para una mayor confianza en el ejecutivo. Mientras que un desempeño positivo en la seguridad ciudadana influye de manera positiva y significativa en la confianza tanto en el ejecutivo como en las municipalidades, lo que sugiere que la ciudadanía percibe esta problemática como una responsabilidad conjunta entre los Gobiernos nacionales y locales. Segovia et al. (2008) respaldan estos resultados argumentando que los ciudadanos emiten sus juicios de confianza sobre la base de una evaluación de la capacidad de la institución para realizar las acciones esperadas por la ciudadanía, y la voluntad de esta para buscar el bien común. Sin embargo, los resultados muestran que el desempeño en materia educacional y de salud tiene una incidencia significativa solo en la confianza de los gobiernos locales, mientras que un buen desempeño económico solo incrementa la confianza en el ejecutivo. Lo anterior está en sintonía con la actual institucionalidad en el país, en la cual la gestión y responsabilidad de la educación y salud pública recae fuertemente en las municipalidades. Un buen desempeño económico que afecte de forma positiva la confianza institucional del Gobierno responde al argumento de que la confianza en las instituciones se correlaciona con el ciclo económico (Gallup et al., 2011).

Tabla 5. Estimaciones para la confianza en instituciones de gobernanza nacional y local

\begin{tabular}{|c|c|c|}
\hline Variables & Ejecutivo & Municipalidad \\
\hline \multicolumn{3}{|c|}{ Socioeconómico } \\
\hline \multirow{2}{*}{ EDU } & $-0,014$ & $-0,002$ \\
\hline & $(0,010)$ & $(0,010)$ \\
\hline \multirow{2}{*}{ EDAD } & 0,003 & $-0,002$ \\
\hline & $(0,003)$ & $(0,003)$ \\
\hline \multirow{2}{*}{ ECV } & $-0,086$ & $-0,079$ \\
\hline & $(0,076)$ & $(0,073)$ \\
\hline \multirow{2}{*}{ MEST } & $-0,055$ & 0,015 \\
\hline & $(0,079)$ & $(0,076)$ \\
\hline \multirow{2}{*}{ INDG } & 0,222 & 0,054 \\
\hline & $(0,146)$ & $(0,179)$ \\
\hline \multirow{2}{*}{$O C P$} & $-0,153^{* *}$ & $-0,145^{*}$ \\
\hline & $(0,074)$ & $(0,074)$ \\
\hline \multirow{2}{*}{ URB } & 0,100 & $-0,055$ \\
\hline & $(0,113)$ & $(0,105)$ \\
\hline \multirow{2}{*}{ CENT } & 0,042 & $0,189 * *$ \\
\hline & $(0,084)$ & $(0,081)$ \\
\hline \multirow{2}{*}{ NOR } & $-0,357^{* * *}$ & $-0,216^{*}$ \\
\hline & $(0,123)$ & $(0,120)$ \\
\hline \multirow{2}{*}{ TMM } & $-0,123^{* *}$ & 0,072 \\
\hline & $(0,051)$ & $(0,055)$ \\
\hline \multicolumn{3}{|c|}{ Democracia } \\
\hline \multirow{2}{*}{ RSP.IP } & 0,042 & $0,091^{* * *}$ \\
\hline & $(0,026)$ & $(0,027)$ \\
\hline
\end{tabular}


Continúa Tabla 5. Estimaciones para la confianza en instituciones de gobernanza nacional y local

\begin{tabular}{|c|c|c|}
\hline Variables & Ejecutivo & Municipalidad \\
\hline \multirow{2}{*}{ C.ELEC } & $0,240^{* * *}$ & $0,287^{* * *}$ \\
\hline & $(0,032)$ & $(0,034)$ \\
\hline \multicolumn{3}{|c|}{ Desempeño } \\
\hline \multirow{2}{*}{ MEDIC } & $-0,013$ & $0,174^{* * *}$ \\
\hline & $(0,027)$ & $(0,027)$ \\
\hline \multirow{2}{*}{ PENPUB } & 0,015 & 0,036 \\
\hline & $(0,023)$ & $(0,024)$ \\
\hline \multirow{2}{*}{ AP. PRES } & $0,633^{* * *}$ & 0,023 \\
\hline & $(0,062)$ & $(0,056)$ \\
\hline \multirow{2}{*}{ SEGCIU } & $0,223^{* * *}$ & $0,125^{* * *}$ \\
\hline & $(0,0408)$ & $(0,046)$ \\
\hline \multirow{2}{*}{ EDUC } & $-0,015$ & $0,117^{* *}$ \\
\hline & $(0,057)$ & $(0,056)$ \\
\hline \multirow{2}{*}{ ECON } & $0,224^{* * *}$ & 0,075 \\
\hline & $(0,065)$ & $(0,064)$ \\
\hline \multicolumn{3}{|c|}{ Participación } \\
\hline \multirow{2}{*}{ REUPOL } & 0,096 & 0,078 \\
\hline & $(0,125)$ & $(0,164)$ \\
\hline \multirow{2}{*}{ REUMUN } & 0,050 & 0,234 \\
\hline & $(0,169)$ & $(0,159)$ \\
\hline \multicolumn{3}{|c|}{ Corrupción } \\
\hline \multirow{2}{*}{ DESCORR } & 0,050 & 0,016 \\
\hline & $(0,038)$ & $(0,041)$ \\
\hline \multirow{2}{*}{ SOBOR } & 0,007 & 0,094 \\
\hline & $(0,159)$ & $(0,168)$ \\
\hline \multicolumn{3}{|c|}{ Umbrales } \\
\hline \multirow{2}{*}{ Constant cuT1 } & $2,727^{* * *}$ & $1,910 * * *$ \\
\hline & $(0,368)$ & $(0,357)$ \\
\hline \multirow{2}{*}{ Constant cuT2 } & $3,219 * * *$ & $2,333^{* * *}$ \\
\hline & $(0,379)$ & $(0,361)$ \\
\hline \multirow{2}{*}{ Constant cut3 } & $3,659 * * *$ & $2,857^{* * *}$ \\
\hline & $(0,387)$ & $(0,368)$ \\
\hline \multirow{2}{*}{ Constant cut4 } & $4,404^{* * *}$ & $3,672^{* * *}$ \\
\hline & $(0,397)$ & $(0,376)$ \\
\hline \multirow{2}{*}{ Constant cut5 } & $5,332^{* * *}$ & $4,666^{* * *}$ \\
\hline & $(0,406)$ & $(0,388)$ \\
\hline \multirow{2}{*}{ Constant cut6 } & $6,240 * * *$ & 5,651 *** \\
\hline & $(0,420)$ & $(0,403)$ \\
\hline Observaciones & 932 & 926 \\
\hline
\end{tabular}

Nota: *** estadísticamente significativos al $99 \%$ de confianza;

**estadísticamente significativos al $95 \%$ de confianza;

*estadísticamente significativos al $90 \%$ de confianza. Errores estándar robustos en paréntesis.

Fuente: elaboración propia.
Finalmente, las variables relacionadas con las dimensiones de participación y corrupción no afectan significativamente la confianza en este tipo de instituciones. Cabe agregar que en 2014, periodo cuando se realiza la encuesta, Chile era el segundo país con menor corrupción en América Latina. Este escenario ha cambiado en la actualidad, con hechos de corrupción emergiendo en los últimos años que redujeron fuertemente la aprobación del Gobierno y potencialmente la confianza en las instituciones.

La tabla 6 presenta los resultados para la confianza en instituciones religiosas.

Tabla 6. Estimaciones para la confianza en instituciones religiosas

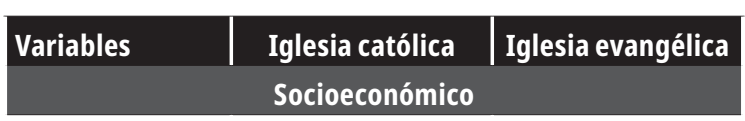

\begin{tabular}{|c|c|c|}
\hline \multirow{2}{*}{ EDU } & $-0,009$ & $-0,014$ \\
\hline & $(0,009)$ & $(0,010)$ \\
\hline \multirow{2}{*}{ EDAD } & $0,007^{* * *}$ & $-0,004$ \\
\hline & $(0,002)$ & $(0,002)$ \\
\hline \multirow{2}{*}{ ECV } & $-0,093$ & 0,046 \\
\hline & $(0,063)$ & $(0,067)$ \\
\hline \multirow{2}{*}{ OCP } & $-0,192^{* * *}$ & 0,033 \\
\hline & $(0,066)$ & $(0,069)$ \\
\hline \multirow{2}{*}{ RELG } & $0,992^{* * *}$ & $0,612^{* * *}$ \\
\hline & $(0,110)$ & $(0,111)$ \\
\hline \multirow{2}{*}{ MEST } & 0,109 & 0,052 \\
\hline & $(0,067)$ & $(0,069)$ \\
\hline \multirow{2}{*}{ INDG } & $-0,237$ & 0,046 \\
\hline & $(0,165)$ & $(0,165)$ \\
\hline \multirow{2}{*}{ URB } & $-0,124$ & 0,006 \\
\hline & $(0,105)$ & $(0,108)$ \\
\hline \multirow{2}{*}{ CENT } & $-0,201^{* * *}$ & $-0,172^{* *}$ \\
\hline & $(0,078)$ & $(0,080)$ \\
\hline \multirow{2}{*}{ NOR } & $-0,354^{* * *}$ & $-0,278^{* *}$ \\
\hline & $(0,115)$ & $(0,112)$ \\
\hline \multirow{2}{*}{ TMM } & $-0,004$ & $-0,036$ \\
\hline & $(0,044)$ & $(0,047)$ \\
\hline \multicolumn{3}{|c|}{ Democracia } \\
\hline \multirow{2}{*}{ C.ELE } & $0,130 * * *$ & $0,116^{* * *}$ \\
\hline & $(0,024)$ & $(0,025)$ \\
\hline \multicolumn{3}{|c|}{ Desempeño } \\
\hline \multirow{2}{*}{ PENPUB } & $0,038^{* *}$ & 0,029 \\
\hline & $(0,019)$ & $(0,021)$ \\
\hline
\end{tabular}


Continuación Tabla 6. Estimaciones para la confianza en instituciones religiosas

\begin{tabular}{|c|c|c|}
\hline Variables & Iglesia católica & Iglesia evangélica \\
\hline \multicolumn{3}{|c|}{ Participación } \\
\hline \multirow{2}{*}{ REUREL } & 0,0121 & $0,247^{* \star *}$ \\
\hline & $(0,033)$ & $(0,035)$ \\
\hline \multicolumn{3}{|c|}{ Valórico } \\
\hline \multirow{2}{*}{ IGUAL } & $0,017^{*}$ & $-0,014$ \\
\hline & $(0,010)$ & $(0,010)$ \\
\hline \multirow{2}{*}{$\mathrm{ABOR}$} & $-0,063$ & $-0,149$ ** \\
\hline & $(0,070)$ & $(0,073)$ \\
\hline \multicolumn{3}{|c|}{ Umbrales } \\
\hline \multirow{2}{*}{ Constant cuT1 } & $0,440^{*}$ & 0,137 \\
\hline & $(0,239)$ & $(0,260)$ \\
\hline \multirow{2}{*}{ Constant cuT2 } & $0,691^{* * *}$ & $0,513^{*}$ \\
\hline & $(0,241)$ & $(0,262)$ \\
\hline \multirow{2}{*}{ Constant cut3 } & $0,997^{* * *}$ & $0,871^{* * *}$ \\
\hline & $(0,242)$ & $(0,264)$ \\
\hline \multirow{2}{*}{ Constant cut 4} & $1,417^{* * *}$ & $1,357^{* * *}$ \\
\hline & $(0,245)$ & $(0,267)$ \\
\hline \multirow{2}{*}{ Constant cut5 } & $2,078^{* * *}$ & $1,965^{* * *}$ \\
\hline & $(0,248)$ & $(0,272)$ \\
\hline \multirow{2}{*}{ Constant cut6 } & $2,679^{* * *}$ & $2,385^{* * *}$ \\
\hline & $(0,251)$ & $(0,271)$ \\
\hline Observaciones & 1,163 & 1,063 \\
\hline
\end{tabular}

Nota: *** estadísticamente significativos al $99 \%$ de confianza; **estadísticamente significativos al $95 \%$ de confianza;

*estadísticamente significativos al $90 \%$ de confianza. Errores estándar robustos en paréntesis.

Fuente: elaboración propia.

Acerca de las variables socioeconómicas y de localización, se tienen mayores niveles de confianza tanto en la iglesia católica, como evangélica de los habitantes del sur del país, una tendencia que se fundamenta en una cultura más conservadora de la zona sur. La confianza en ambas iglesias también es más alta entre las personas que reportan ser mestizos. Con respecto a la edad, se tienen resultados dispares. Mientras que para la iglesia católica la confianza se incrementa con la edad, se presenta lo opuesto para los niveles de confianza en la iglesia evangélica. Este fenómeno podría estar relacionado con el aumento que ha experimentado la población evangélica en el país y la baja importante de las personas que profesan la religión católica en la última década, situación que cambia la composición etaria y hace más longeva a la comunidad católica respecto a la comunidad evangélica.

Variables relacionadas con la percepción de la democracia, el desempeño y la participación también influyen positiva y significativamente en la confianza de este tipo de instituciones. Por ejemplo, las personas que confían más en el Sistema Electoral manifiestan mayor confianza en este tipo de instituciones. Asimismo, si las personas piensan que sus intereses y pensamientos están siendo acogidos por la institución religiosa, esto incrementa la confianza institucional en la iglesia católica, así como la asistencia a reuniones promueve mayores niveles de confianza en la iglesia evangélica. Esto último podría ser consecuencia de canales de participación distintos entre religiones, donde la evidencia indica una mayor cercanía y representatividad de sus miembros en la iglesia evangélica, con una estructura que fomenta mucho más la participación de sus feligreses.

Cabe agregar que puede resultar inadecuado estudiar aspectos democráticos y de desempeño para el caso de las instituciones religiosas, ya que en particular estas no deben cumplir propuestas de la ciudadanía ni generar satisfacción con su actuar; más bien, la afección a estas radica en concepciones morales y de fe que comulguen con las doctrinas teológicas que tienen como base estas instituciones. Dada esta naturaleza de las instituciones religiosas, para las estimaciones del modelo de confianza institucional se incorporan a la discusión variables de orden valórico relacionadas con la aceptación de la homosexualidad y el aborto. Los resultados muestran que las personas que aprueban la práctica del aborto en una situación de riesgo vital de la madre confían menos en la iglesia evangélica, fenómeno que se sustenta en una ideología mucho más conservadora en relación con el aborto de esta comunidad religiosa. Adicionalmente, las personas que sostienen que están de acuerdo con el matrimonio homosexual confían más en la iglesia católica, aunque esta relación es significativa solo al $10 \%$. Lo anterior podría relacionarse con la particularidad de una comunidad católica que simpatiza con la religión, pero que se encuentra mucho 
menos comprometida con esta y es más abierta a una idea globalizada, lo cual genera discrepancias entre la doctrina católica y lo que piensan sus simpatizantes. En contraste, la iglesia evangélica está fundamentada en una concepción mucho más unificada y en sintonía con sus creencias.

Por último, un ámbito relevante que podría afectar los niveles de confianza en las instituciones religiosas anteriores, y que no fue posible abordar en la presente investigación a través de la encuesta Latin American Public Opinion Project (LAPOP), es el grado de conocimiento o victimización en casos de abuso sexual infantil cometidos por personeros eclesiásticos. En este sentido, la evidencia encontrada por Rossetti (1995) sugiere la existencia de menores niveles de confianza hacia la iglesia católica, y su relación con Dios, entre adultos víctimas de abuso sexual perpetrado por clérigos durante su infancia.

\section{Conclusiones}

La confianza institucional es relevante para la sociedad, ya que esta es un determinante fundamental para que pueda existir un buen gobierno y desarrollo económico, además de promover condiciones que ayuden al surgimiento de una democracia sana y su buen funcionamiento. Chile, a pesar de ser uno de los países menos corruptos de Latinoamérica, ha mostrado una tendencia a la desafección política y desconfianza institucional en los últimos años, evidenciada por una baja participación cívica y organizacional, potencialmente debido a los hechos de corrupción que se han revelado recientemente en las instituciones. Lo anterior ha generado una disconformidad generalizada con los organismos que representan a la población, y así ha quedado en tela de juicio su real capacidad de canalizar el pensar colectivo. Esta investigación analizó un conjunto de variables que podrían explicar este fenómeno, haciendo énfasis en factores socioeconómicos, de percepción sobre la democracia, de desempeño institucional, participación civil y corrupción. Se estudió, además, la confianza institucional no solo en instituciones de carácter gubernamental y político, sino también religioso. Para tal propósito, se estimó un modelo probit ordenado haciendo uso de los datos proporcionados por la encuesta LAPOP de 2014.

Los resultados muestran una visión más crítica materializada en una desconfianza mayor desde los habitantes que residen en la zona norte del país, en general, y las comunidades de pueblos originarios, particularmente en las instituciones de seguridad; esto último es probable que esté fundamentado por el conflicto mapuche. Asimismo, se encuentra un efecto positivo y significativo de variables asociadas a la legitimidad de la democracia como sistema político sobre los niveles de confianza en instituciones políticas y de gobierno. Destaca aquí la confianza inherente en las Fuerzas Armadas por parte de posiciones más conservadoras y determinadas por una comunidad más identificada con el régimen militar, cuyos valores antidemocráticos parecen persistir aún en el país.

Las variables de desempeño también muestran una relación positiva y significativa con la confianza institucional, lo que implica que la ciudadanía premie o castigue la labor de las instituciones en función de sus compromisos y metas. Como lo destacan Stoyan et al. (2016), este desempeño, que no se remite solo al ámbito económico, puede afectar significativamente la confianza institucional, en especial en democracias emergentes o no plenamente establecidas como la chilena. Diferencias entre instituciones del plano ejecutivo y municipal sugieren responsabilidades dispares, donde al ejecutivo se le percibe responsable de la economía y a la municipalidad de la provisión de bienes públicos locales, como son la educación y la salud.

Para el caso de la participación, los resultados sugieren que las instituciones que cuentan con instancias para una interacción con los representantes se ven favorecidas en sus niveles de confianzas. Esto se evidencia con mayor claridad en el caso de las instituciones religiosas y políticas, y se destaca la importancia de los mecanismos de retroalimentación y trabajo con participación de la comunidad.

Por otra parte, se encontró que la corrupción afecta los niveles de confianza, pero este efecto 
varía por tipo de institución. En particular, para el caso de las Fuerzas Armadas, los resultados concluyen una relación positiva entre la confianza institucional y variables ligadas a la corrupción, lo que sugiere que ambas instituciones podrían verse como sustitutas en situaciones de descontento social. Aunque no es posible con los datos verificar plenamente esta hipótesis, en Chile aún existe una visión compartida de la existencia de prerrogativas de las Fuerzas Armadas por encima del poder político (Garretón, 2007). Esta visión es respaldada por hechos recientes, por ejemplo, la reticencia del Gobierno de turno para llamar a las Fuerzas Armadas en situaciones de caos público durante el terremoto de 2010 en Chile (Baeza, 2010). Esta línea de discusión merece mucha más atención en trabajos futuros.

En el caso particular de las instituciones religiosas, se hizo una modificación del modelo base para profundizar en temas valóricos como el aborto legal y el matrimonio igualitario. Los resultados evidencian niveles de confianza menores en la iglesia evangélica por parte de personas que están de acuerdo con el aborto en caso de peligro de la madre, y niveles de confianza mayores en la iglesia católica en las personas que están de acuerdo con el matrimonio homosexual. Lo anterior sugiere que la comunidad evangélica es percibida con una postura mucha más rígida y en sintonía con su doctrina en cuanto a los aspectos valóricos, en tanto los católicos, al ser una comunidad mucho más grande y diversa, se percibe como más heterogénea en términos valóricos.

Dentro de las limitaciones de la presente investigación, es posible señalar que el conjunto de datos utilizado no captura la victimización por abuso sexual infantil cometido por representantes de instituciones religiosas, ni hechos recientes de corrupción y de gestión deficiente reportados en instituciones chilenas, e. g., fraude financiero dentro de Carabineros, violaciones a los derechos humanos en el interior del Servicio Nacional de Menores (Sename), organismo dependiente del Ministerio de Justicia. Por ende, el estudio del potencial efecto negativo de tales eventos sobre los niveles de confianza en Chile es un flanco de investigación futura.

\section{Agradecimientos}

La presente investigación fue desarrollada en el proyecto grupo de investigación GI 160317/EF Análisis económico sectorial aplicado, de la Universidad del Bío-Bío, Chile. Los autores agradecen a Vanderbilt University por proveer libremente los datos de la encuesta LAPOP. Adicionalmente, agradecen los comentarios de los evaluadores anónimos que contribuyeron a mejorar de forma substancial este artículo. Todos los resultados y las interpretación de los datos son estricta responsabilidad de los autores.

\section{Referencias}

Ahlerup, P., Olsson, O. y Yanagizawa, D. (2009). Social capital vs. institutions in the growth process. European Journal of Political Economy, 25(1), 1-14.

Aker, J. C. (2007). Social networks and household welfare in Tanzania: Working together to get out of poverty. SSRN Scholarly Paper, N. ${ }^{\circ}$ ID 995941. Rochester, Estados Unidos: Social Science Research Network.

Almond, G. A. y Verba, S. (2015). The civic culture: Political attitudes and democracy in five nations. Princenton, Estados Unidos: Princeton University Press.

Anderson, C. J. y Tverdova, Y. V. (2001). Winners, losers, and attitudes about government in contemporary democracies. International Political Science Review, 22(4), 321-338.

Armah-Attoh, D., Gyimah-Boadi, E. y Chikwanha, A. B. (2007). Corruption and institutional trust in Africa: Implications for democratic development. Afrobarometer Working Paper, (81), 1-34.

Baeza, R. (2010). Carnaval perverso: terremoto + tsunami y saqueos en el Chile de 2010. Sociedad Hoy, (19), 53-69.

Bentham, J. (1989). First principles preparatory to constitutional conde. Oxford: Clarendon Press.

Beramendi, M., Delfino, G. y Zubieta, E. (2016). Confianza institucional y social: una relación insoslayable. Acta de Investigación Psicológica, 6(1), 2286-2301.

Bowler, S. y Karp, J. A. (2004). Politicians, scandals, and trust in government. Political Behavior, 26(3), 271-287.

Coleman, J. S. (1988). Social capital in the creation of human capital. American Journal of Sociology, 94(1), 95-120.

Consejo de Transparencia. (2017). VIII Estudio nacional de transparencia. Santiago de Chile: Dirección de Estudios. 
Corbacho, A., Philipp, J. y Ruiz-Vega, M. (2015). Crime and erosion of trust: Evidence for Latin America. World Development, (70), 400-415.

Cornú, L. (1999). La confianza en las relaciones pedagógicas. En G. Frigerio, M. Poggi y D. Korinfeld (Comps.), Construyendo un saber sobre el interior de la escuela (pp. 19-26). Buenos Aires: Novedades Educativas.

Easterly, W., Ritzen, J. y Woolcock, M. (2006). Social cohesion, institutions, and growth. Economics \& Politics, 18(2), 103-120.

Easton, D. (1965). A systems analysis of political life. Nueva York, Estados Unidos: Wiley.

Echebarría, K. (2007). Corrupción: Un análisis a través de indicadores de gobernabilidad. Washington, D. C., Estados Unidos: Inter-American Development Bank.

Fukuyama, F. (1995). Trust: The social virtues and the creation of prosperity. Nueva York: Free Press Paperbacks.

Garretón, M. A. (2007). Del postpinochetismo a la sociedad democrática. Santiago de Chile, Chile: Prometeo.

Golder, M. (2005). Democratic electoral systems around the world, 1946-2000. Electoral Studies, 24(1), 103-121.

Gordon, S. (2006). Confianza, reciprocidad y asociatividad: ¿relación indispensable para el desempeño institucional? Estudios Sociológicos, 24(71), 397-421.

Granovetter, M. (1985). Economic action and social structure: The problem of embeddedness. American Journal of Sociology, 91(3), 481-510.

Hommerich, C. (2012). Trust and subjective well-being after the great east Japan earthquake, tsunami and nuclear meltdown: Preliminary results. International Journal of Japanese Sociology, 21(1), 46-64.

Horváth, R. (2013). Does trust promote growth? Journal of Comparative Economics, 41(3), 777-788.

Hudson, J. (2006). Institutional trust and subjective well-being across the EU. Kyklos, 59(1), 43-62.

Kliksberg, B. (1999). Social capital and culture: Master keys to development. Cepal Review, (69), 83-102.

Knack, S. y Keefer, P. (1997). Does Social Capital Have an Economic Payoff? A Cross-Country Investigation. The Quarterly Journal of Economics, 112(4), 1251-1288.

Knight, J. (1998). The bases of cooperation: Social norms and the rule of law. Journal of Institutional and Theoretical Economics, 154(4), 354-373.

Proyecto de Opinión Publica en América Latina (LAPOP). (2010). Proyecto de Opinión Pública de América Latina. Departamento de Ciencia Política, Vanderbilt University. Recuperado de https://www.vanderbilt.edu/ lapop-espanol/

Luhmann, N. (2005). Confianza. Barcelona, España: Anthropos.
Mayer, R. C., Davis, J. H. y Schoorman, F. D. (1995). An integrative model of organizational trust. Academy of Management Review, 20(3), 709-734.

Montero, J. R., Gunther, R., Torcal, M. y Menezo, J. C. (1998). Actitudes hacia la democracia en España: legitimidad, descontento y desafección. Reis: Revista Española de Investigaciones Sociológicas, (83), 9-49.

Morales, M. (2008). Evaluando la confianza institucional en Chile: una mirada desde los resultados LAPOP. Revista de Ciencia Política, 28(2), 161-186.

Morris, S. D. y Klesner, J. L. (2010). Corruption and trust: Theoretical considerations and evidence from Mexico. Comparative Political Studies, 43(10), 1258-1285.

Norris, P. (1999). Critical citizens: Global support for democratic government. Oxford, Inglaterra: Oxford University Press.

North, D. C. (1990). Institutions, institutional change and economic performance. Cambridge, Inglaterra: Cambridge University Press.

Nye, J. S., Zelikow, P. D. y King, D. C. (1997). Why people don't trust government. Cambridge, Inglaterra: Harvard University Press.

Pharr, S. J. y Putnam, R. D. (Eds.). (2000). Disaffected democracies: What's troubling the trilateral countries? Princeton, Estados Unidos: Princeton University Press.

Putnam, R. D., Leonardi, R. y Nanetti, R. Y. (1993). Making democracy work: Civic traditions in modern Italy. Princeton, Estados Unidos: Princeton University Press.

Rosas, G. (2010). Trust in elections and the institutional design of electoral authorities: Evidence from Latin America. Electoral Studies, 29(1), 74-90.

Rossetti, S. J. (1995). The impact of child sexual abuse on attitudes toward god and the catholic church. Child Abuse \& Neglect, 19(12), 1469-1481.

Sarsfield, R. y Echegaray, F. (2005). Opening the black box: How satisfaction with democracy and its perceived efficacy affect regime preference in Latin America. International Journal of Public Opinion Research, 18(2), 153-173.

Segovia, C., Haye, A., González, R., Manzi, J. y Carvacho, H. (2008). Confianza en instituciones políticas en Chile: un modelo de los componentes centrales de juicios de confianza. Revista de Ciencia Política, 28(2), 39-60.

Stevenson, B. y Wolfers, J. (2011). Trust in public institutions over the business cycle. American Economic Review, 101(3), 281-287.

Stoyan, A. T., Niedzwiecki, S., Morgan, J., Hartlyn, J. y Espinal, R. (2016). Trust in government institutions: 
The effects of performance and participation in the Dominican Republic and Haiti. International Political Science Review, 37(1), 18-35.

Torcal, M. (2000). Partidos y desafección política. Desarrollo Humano e Institucional en América Latina (DHIAL), 14.

Westlund, H. y Adam, F. (2010). Social capital and economic performance: A meta-analysis of 65 studies. European Planning Studies, 18(6), 893-919.
Wilson, W. J. (1996). When work disappears: The world of the new urban poor. Nueva York, Estados Unidos: Knopf Doubleday.

Woolcock, M. y Narayan, D. (2000). Social capital: Implications for development theory, research, and policy. The World Bank Research Observer, 15(2), 225-249. 